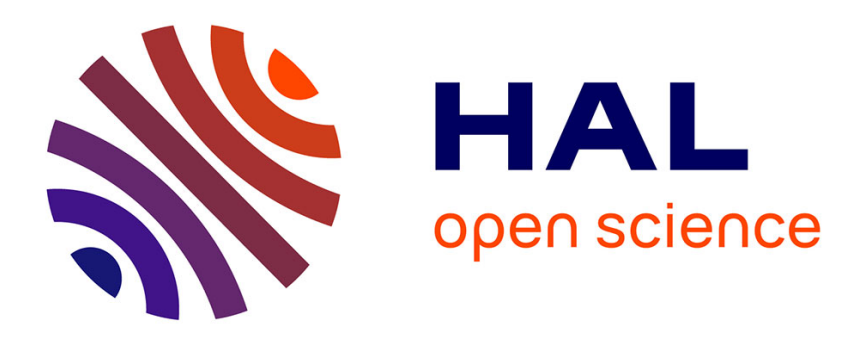

\title{
Analog electro-optical interface for spectral analysis
}

G. Labrunie

\section{To cite this version:}

G. Labrunie. Analog electro-optical interface for spectral analysis. Revue de Physique Appliquée, 1975, 10 (4), pp.213-219. 10.1051/rphysap:01975001004021300 . jpa-00243906

\section{HAL Id: jpa-00243906 https://hal.science/jpa-00243906}

Submitted on 1 Jan 1975

HAL is a multi-disciplinary open access archive for the deposit and dissemination of scientific research documents, whether they are published or not. The documents may come from teaching and research institutions in France or abroad, or from public or private research centers.
L'archive ouverte pluridisciplinaire HAL, est destinée au dépôt et à la diffusion de documents scientifiques de niveau recherche, publiés ou non, émanant des établissements d'enseignement et de recherche français ou étrangers, des laboratoires publics ou privés. 


\author{
Classification \\ Physics Abstracts \\ $2.450-8.540$
}

\title{
ANALOG ELECTRO-OPTICAL INTERFACE FOR SPECTRAL ANALYSIS (*)
}

\author{
G. LABRUNIE
}

\author{
C. E. N. G.-L. E. T. I., B. P. 85, Centre de Tri, 38041 Grenoble Cedex, France
}

(Reçu le 22 juillet 1974, révisé le 6 février 1975, accepté le 25 février 1975)

\begin{abstract}
Résumé. - Un relais électro-optique analogique a été mis au point; il met en œuvre l'effet de champ bien connu qui induit une modulation d'indice dans une couche mince homéotrope de cristal liquide nématique soumise à un champ électrique alternatif. Un tel dispositif peut présenter en temps réel 64 échantillons analogiques d'un signal électrique sur un montage d'optique cohérente en quelques millisecondes. Avec ce relais, des signaux électriques comme les électroencéphalogrammes, les vibrations de moteurs ou les signaux SONAR peuvent subir une analyse spectrale en temps réel par transformée de Fourier optique.

Abstract. - An analog electro-optical interface has been studied, which uses the well known field effect of index modulation in thin homeotropic layers of nematic liquid crystal, achieved under a. c. electric field. Such a device is able to present in real-time 64 analog samples of an electric signal in a few milliseconds on a coherent optical set. By means of this interface, electric signals such as electro encephalograms, engine vibrations or SONAR signals can be spectrally analyzed in real-time by optical Fourier transform.
\end{abstract}

1. Introduction. - Coherent optics is known to be the most powerful means to perform information processing $[1,2]$ and that means is instantaneous by comparison with all other techniques which can be used.

But there is a need for analog interfaces able to present, in real-time, the information to be processed into the coherent optical beam in a useful form : spatial variation of phase, or amplitude, or else polarization state of the electromagnetic wave.

A lot of such interfaces have been realized in recent years, but they are generally optically written. In these photoconductor-electro optic medium sandwiches, the electro-optical medium can be a K. D*. P. crystal [3], a P. L. Z. T. ceramic [4], or a nematic liquid crystal used in its dynamic scattering mode $[5,6]$. Such photosensitive-electro-optic materials as bismuth silicate $\left(\mathrm{Bi}_{12} \mathrm{SiO}_{20}\right)$ [7, 8] have recently led to very impressive displays.

But in the general case, the information to be processed is an electric one. This is the reason why we have studied an analog electro-optical device using

(*) Presented at the Vth International Liquid Crystal Conference, Stockholm, 17-21 june 1974.

REVUE DE PHYSIQUE APPLIQUÉE. - T. 10, No 4, JULLLET 1975 the well known field effect of index modulation in a thin homeotropic layer of nematic liquid crystal with negative dielectric anisotropy $(\varepsilon a<0)$, achieved under a. c. electric field.

The information is written by means of electric fields established between transparent electrodes.

2. Spectral analysis in coherent light. - Following G. W. Stroke [1], let us affirm that the principle of a spectrum analyzer is based on the fact that the amplitude distribution of the light in the diffraction patterns formed by an optical grating is equal to the Fourier transform of the amplitude transmitted through the grating when the grating is illuminated by a plane monochromatic wave. In other words, when the grating contains some information deliberately introduced into it, for instance, then the spectrum formed by optical Fourier transform will immediately provide the spectral distribution of the signal (informations) amplitude variations as a function of the chosen coordinate in the plane of the signal.

In the simple experiment of optical Fourier transform shown figure 1 , we are looking at the amplitude distribution $\mathrm{E}(u, v)$ of the light in the focal plane of the high quality optical system (objective) when a signal is introduced in the object plane of this objective under 
the form of an amplitude transparency function $T(x, y)$. This amplitude distribution is :

$$
E(u, v)=\iint T(x, y) \cdot \exp -2 \pi i(u x+v y) \cdot \mathrm{d} x \cdot \mathrm{d} y
$$

$E(u, v)$ is the harmonic analysis, or spectral analysis of the signal $T(x, y)$.

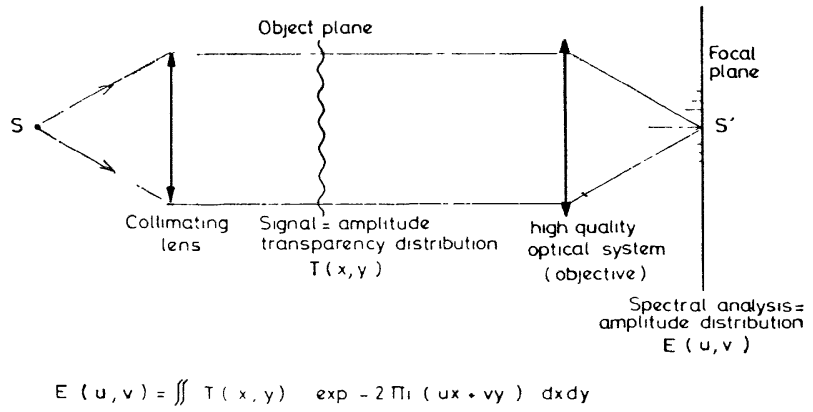

Fig. 1. - The optical Fourier transform.

With any kind of optical detector, we can reach and record only the spectral intensity distribution :

$$
I(u, v)=E(u, v) \cdot E^{*}(u, v)=|E(u, v)|^{2} .
$$

Now, let us consider the case where an electric signal $V(t)$ is spatially recorded and/or displayed as an amplitude transparency function $T(x)$, which is monodimensional. By optical Fourier transform, it will be spectrally analyzed, and the spectral intensity distribution $I(u)=|E(u)|^{2}$ detected in the focal plane will be a spatial display of the spectral intensity distribution $I(v)$ versus frequency $v$ of the signal $V(t)$.

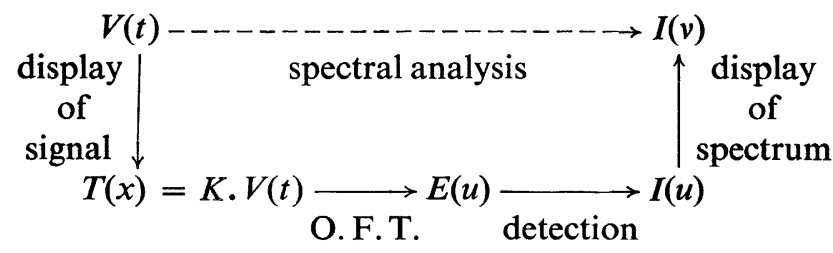

An important feature of optical spectrum analyzers is that they can be designed to perform the spectral analysis simultaneously for many channels in parallel [9].

3. Performing a spectral analysis with the interface. - Figure 2 gives the principle of a spectral analysis performed with the nematic liquid crystal (N. L. C.) interface.

The amplitude of the electric signal $V(t)$ to be spectrally analyzed (Fig. $2 a$ ) is sampled with a frequency $f_{\mathrm{s}}$ and each sample is quantised on 16 analog levels via an electronic circuitry. The result, shown figure $2 b$, is displayed onto the N. L. C. interface in the following way: each analog sample of $V(t)$ is represented by a discrete value of the amplitude transparency $t(x)$ proportional to $V(t)$, with light valves of width a and spatial period $p$. Therefore, the amplitude

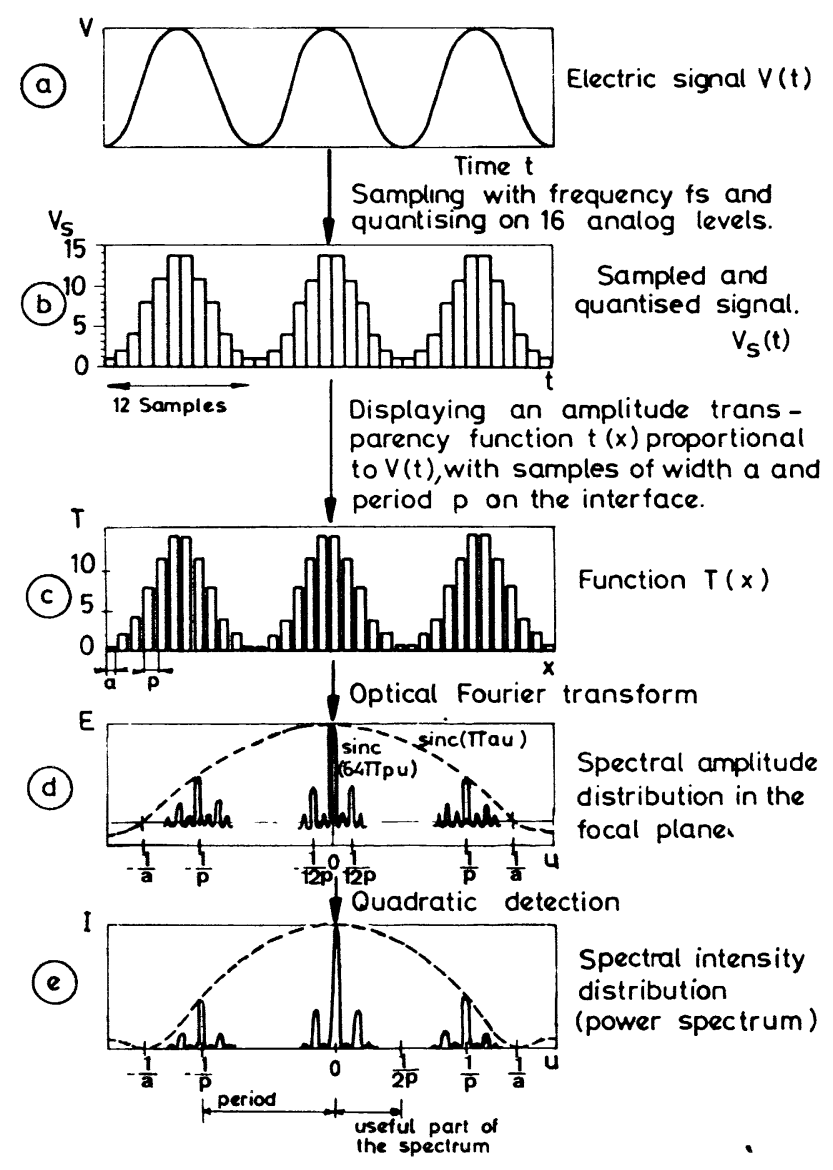

FIG. 2. - Spectral analysis with a nematic liquid crystal interface.

transparency function $T(x)$ displayed onto the interface is the product of 3 terms :

- The function $t(x)$ to be analyzed (quantised);

- The function

$$
\sum_{k=-\infty}^{+\infty} \operatorname{rect}\left(\frac{x}{a}\right) * \delta(x-k p)
$$

(where $*$ is a convolution product and $\sum_{k} \delta(x-k p$ ) the Dirac function spatially recurred with a period $p$ ). This term corresponds to the fact that $t(x)$ is displayed by means of light valves of width $a$ and period $p$.

- The function rect $\left(\frac{x}{64 p}\right)$ due to the fact that the pupil has a finite dimension equal to $64 p$ (because there are 64 samples in the interface).

$$
\text { So : } \begin{aligned}
T(x)=t(x) \times\left[\sum_{k=-\infty}^{+\infty} \operatorname{rect}\left(\frac{x}{a}\right) * \delta\right. & (x-k p)] \times \\
& \times \operatorname{rect}\left(\frac{x}{64 p}\right) .
\end{aligned}
$$

The function $T(x)$ is shown figure $2 c$. By optical Fourier transform, it comes :

$$
\begin{array}{r}
E(u)=e(u) *\left[\sum_{k=-\infty}^{+\infty} \operatorname{sinc}(\pi a u) \times \delta(u-k / p)\right] * \\
* \operatorname{sinc}(64 \pi p u)
\end{array}
$$


where

$$
\operatorname{sinc}(\pi a u) \equiv \frac{\sin \pi a u}{\pi a u}
$$

Hence, what appears in the spectral plane is the convolution product of :

- The spectrum $e(u)$ which we are looking for.

- The Dirac function spatially recurred with a period $1 / p$, times the function sinc $(\pi a u)$ of diffraction of one sample in the interface. This term explains the period $1 / p$ observed in the spectrum, and the amplitude variation of the peaks of diffraction (see Fig. $2 d$ ).

- The function sinc $(64 \pi p u)$ of diffraction of the pupil, which enlarges each peak in the spectrum.

The result of this convolution product is schematically shown figure $2 d$.

By quadratic detection, we observe the intensity spectrum $I(u)=|E(u)|^{2}$ (Fig. 2e) which is a display of the intensity spectrum $I(v)$ of the signal $V(t)$ sampled and quantised.

In $I(u)$ appears clearly (Fig. $2 e$ ) :

- the symmetry of the spectrum (in the experiments, we will look only at one half of it) ;

- the spatial recurrence of period $1 / p$ in the spectrum (sampling gives rise to peaks $\pm 1 / p, \pm 2 / p, \ldots$ );

- the $O$ order due to the fact that the amplitude transparency never has a null mean value.

Obviously, the chosen sinusoïdal function of this example has the simplest spectrum that can be found.

4. Nematic liquid crystal interface and its electronics. - The device (Fig. 3) is made of two glass plates optically flattened, coated with transparent electrodes of indium oxyde. One of these electrodes is not etched, and plays the part of a common electrode. The other one is chemically etched in 64 stripes on which will be displayed the 64 samples of the electric signal to be analyzed. The spacing of the stripes is $100 \mu \mathrm{m}$, each stripe being $80 \mu \mathrm{m}$ wide and $4 \mathrm{~mm}$ long.

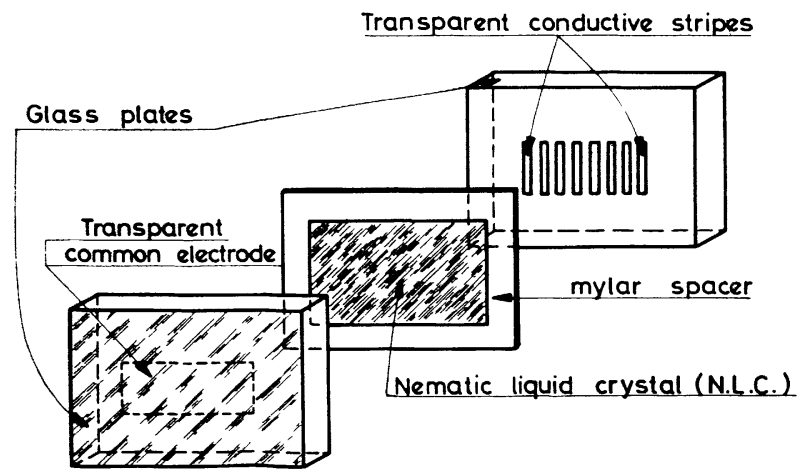

Fig. 3. - Exploded view of the N. L. C. interface.

M. B. B. A. (p-Methoxy benzilidène-p-n-Butylaniline) is introduced between the two plates, under a thickness $L$ of about $8 \mu \mathrm{m}$ obtained with a mylar spacer. The indium oxide electrodes carefully cleaned give rise to an homeotropic structure in such a cell.

Taking advantage of the well known dynamic storage mode [10], the device is addressed sequentially, one sample at a time, by means of a. c. electric fields of about $100 \mathrm{kV} / \mathrm{cm}$. Rise times of a few tens of microseconds for each sample are achieved under these conditions, whereas storage times of 10 to $20 \mathrm{~ms}$ are obtained for such thicknesses within the liquid crystal in a recurrent addressing mode (in our case, for example 25 signal blocks per second).

The signal $V_{\mathrm{e}}$ to be analyzed is fed into a driving electronics, schematically shown figure 4. After a level adjust, the signal is sampled with a frequency ranging from $70 \mathrm{~Hz}$ to $100 \mathrm{kHz}$, and the analog level of each sample is quantised by an analog-digital converter on 4 bits, corresponding to 16 discrete levels. Each block of 64 samples of signal is stored in a 256 digits memory.

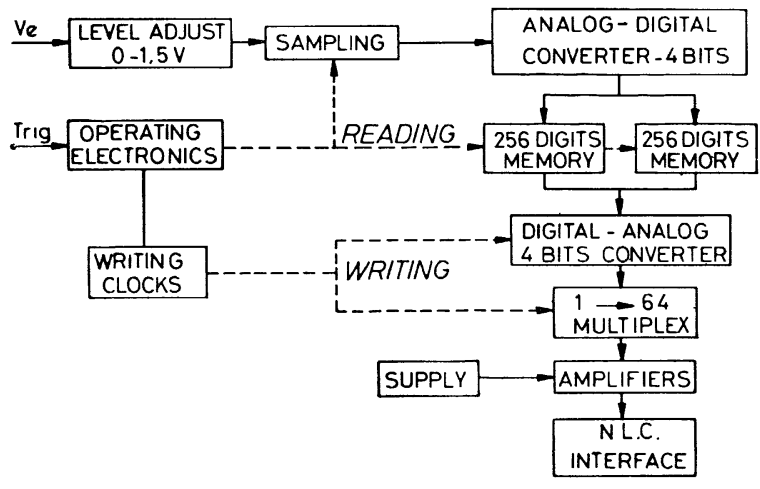

FIG. 4. - Schematic of the operating electronics.

This constitutes the reading part of the electronics, triggered by the signal or by an external reading order.

Then, the 256 digits memory is emptied into a digitalanalog converter, which converts the discrete level of a sample into a pulsewidth, with a conversion law which we will describe later. The 64 pulses are then multiplexed into the operating amplifiers delivering on each stripe of the device an a. c. electric pulse of a fixed value, the width of which is related to the analog level of the corresponding sample.

A second 256 digits memory allows us to read a signal block whereas the first memory is written on the N. L. C. interface, and hence to work in real-time without losing information. It also allows us to compare electric signals which are not contemporary.

Such a driving electronics is characterized by a sampling frequency independent of the displaying speed of informations onto the device.

5. Displaying analog electro-optical informations. It is well known that in the field effect mode, the increase of birefringence $\Delta n(t)$ versus time is approximately exponential for small variations of birefringence [10]. And the exponent is proportional, for a 
given nematic liquid crystal, in a given geometry, to the square pulse voltage, times the pulse width :

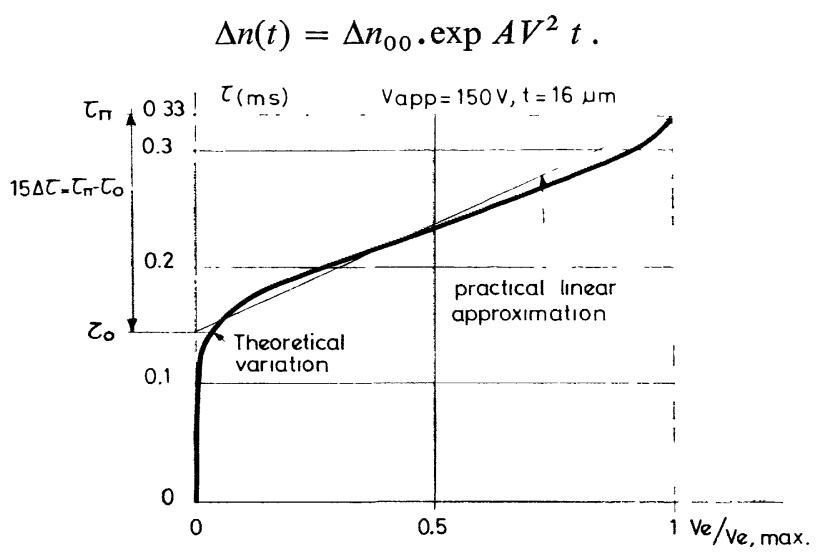

FIG. 5. - Theoretical variation of pulsewidth versus normalized analog level of the signal $V_{\mathrm{e}} / V_{\mathrm{e}}, \max$ for a single addressing.

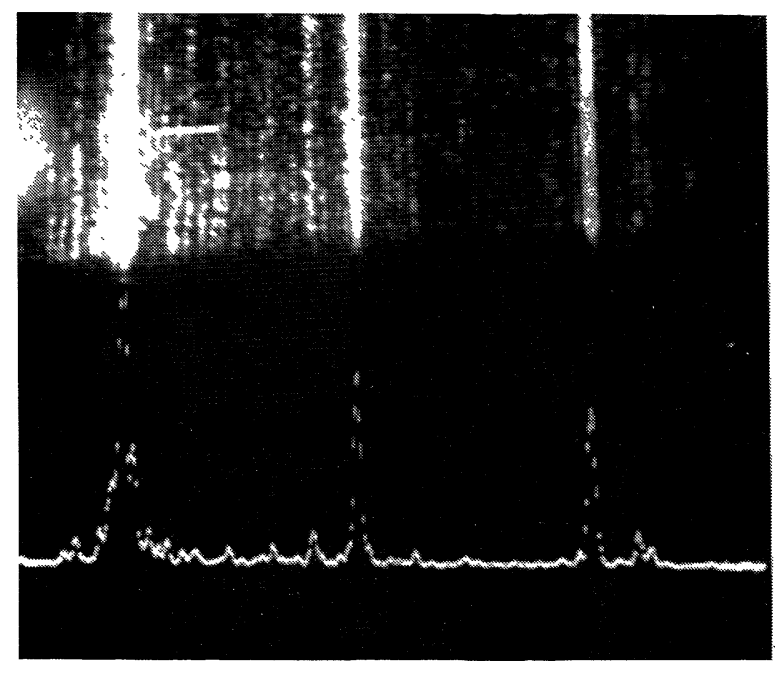

(a)

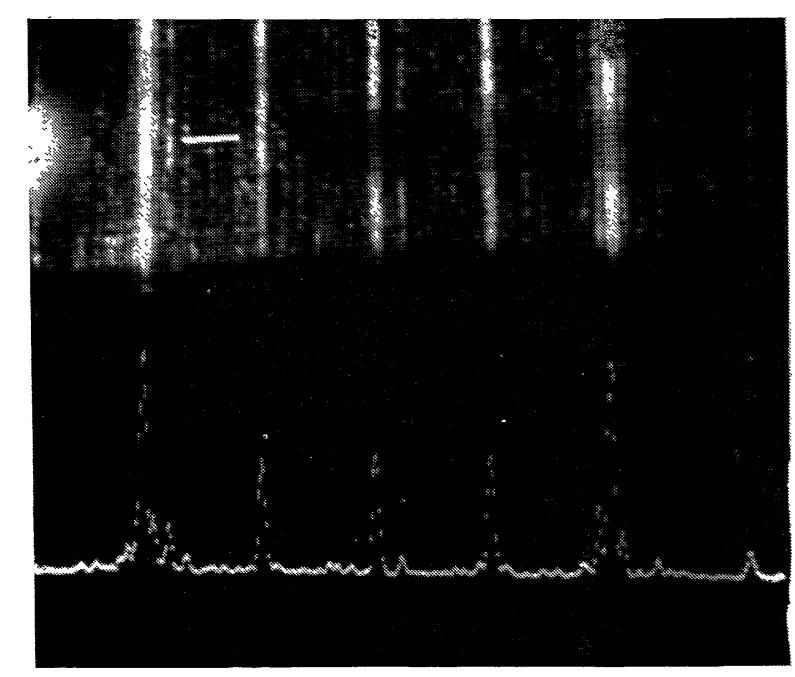

(c)

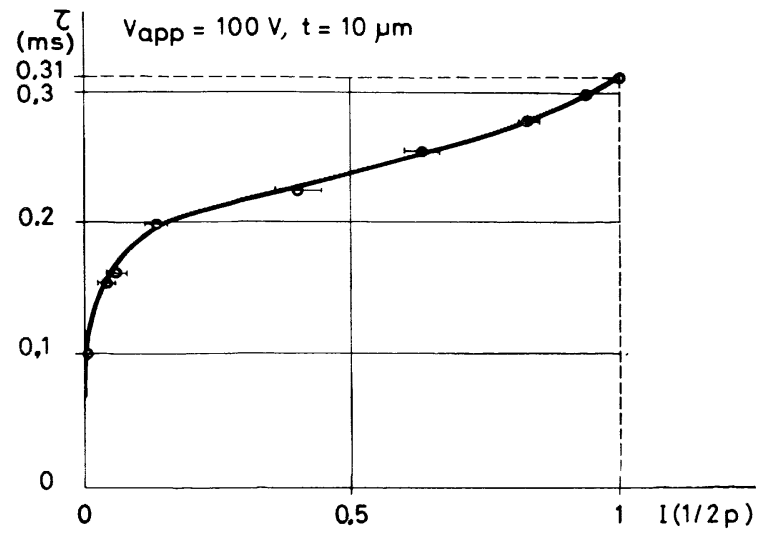

FIG. 6. - Experimental variation of pulsewidth versus normalized analog level $I(1 / 2 p)$ of light intensity diffracted in the order $1 / 2 p$, for a single addressing.

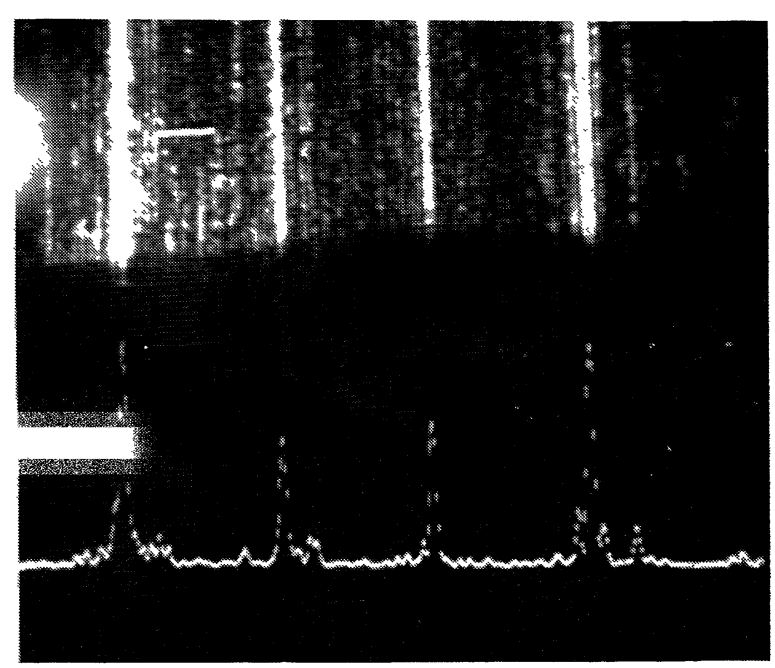

(b)

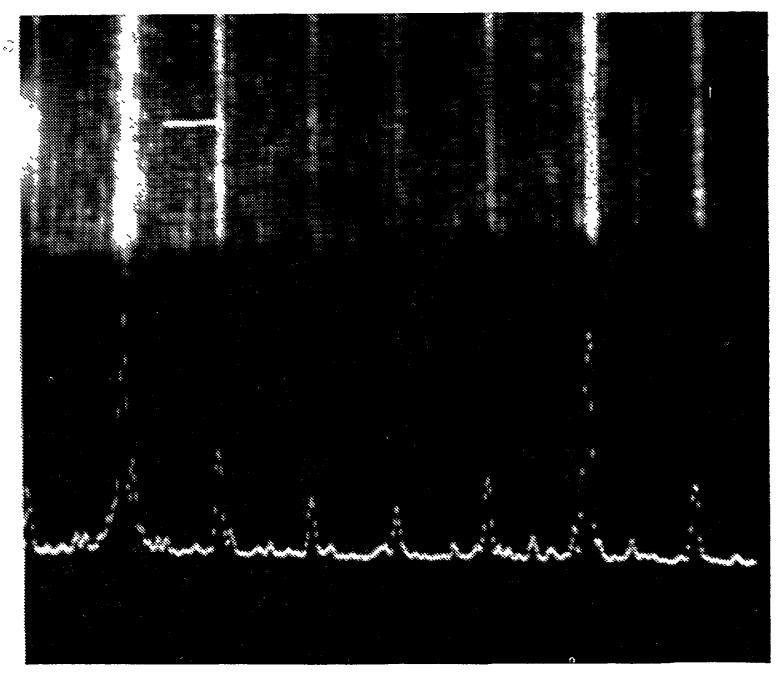

(d)

FIG. 7. - Analyzing various frequencies : for a sampling frequency $f_{\mathrm{s}}$, square functions are displayed, of frequencies : a) $\left.f_{\mathrm{s}} / 2 ; b\right) f_{\mathrm{s}} / 3$;c) $\left.f_{\mathrm{s}} / 4 ; d\right) f_{\mathrm{s}} / 5$.

top : diffraction pattern in the focal plane.

bottom : corresponding spectral distribution. 
So we can control the analog level of $\Delta n(t)$ either by the value of the addressing pulse voltage, or by the value of the pulsewidth. We have experimentally checked the theoretical law.

For the sake of electronic simplicity, we choose the analog control of $\Delta n(t)$ by the pulsewidth $\tau$. Figure 5 shows the theoretical variation of pulsewidth $\tau$ to be applied on a stripe to induce a birefringence giving rise between crossed polarizers to an amplitude transparency proportional to the analog level of the electric signal $V_{\mathrm{e}}$. The practical linear approximation used in the addressing electronics is also shown : the « 0 » level is addressed by a $\tau_{0}$ pulsewidth, and the « 1 » level by a $\tau_{\pi}$ pulsewidth; intermediate levels are

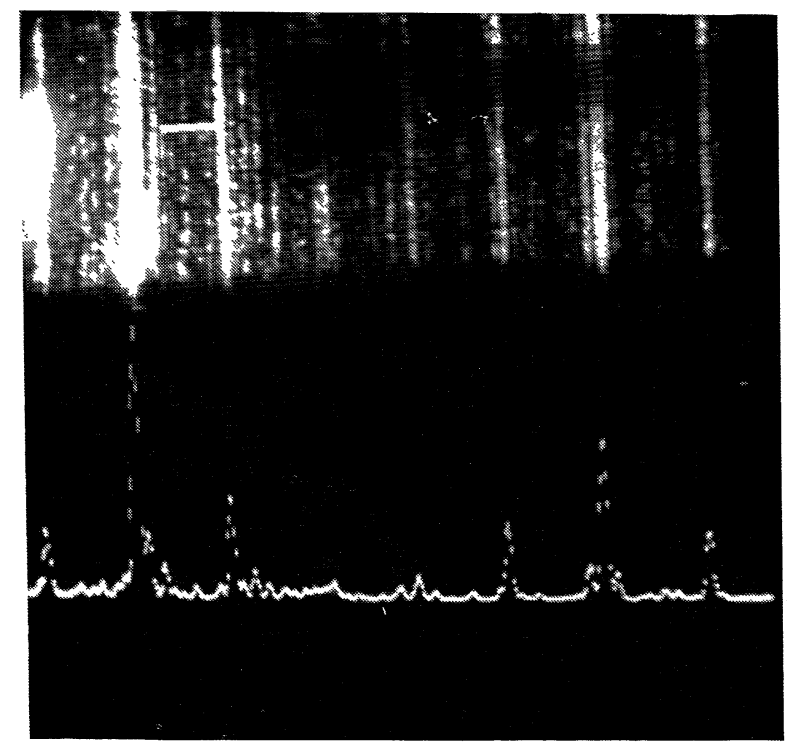

(a)

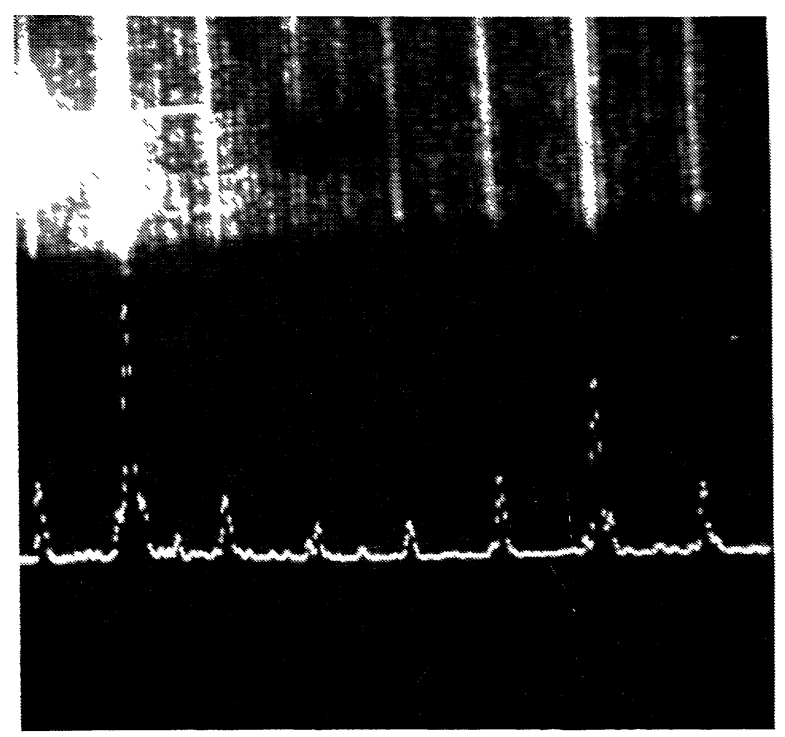

(b) obtained by dividing the $\left(\tau_{\pi}-\tau_{0}\right)$ value into 15 intervals $\Delta \tau$. So, a discrete amplitude of signal $h(0 \leqslant h \leqslant 15)$ will be displayed by means of a $\left(\tau_{0}+h \Delta \tau\right)$ pulsewidth.

Figure 6 confirms the previous theoretical curve: it shows the experimental variation of the normalized analog level $I(1 / 2 p)$ of light intensity diffracted in the order of diffraction $1 / 2 p$ by the grating made of 32 stripes with a $2 p$ spacing addressed by $\tau$ pulsewidths. Fourier transform being a linear transform, it is an experimental verification of the previous theoretical variation of pulsewidth $\tau$ versus the analog level of signal $V_{\mathrm{e}}$, because :

Figure 5: $V_{\mathrm{e}} / V_{\mathrm{e}, \max }$ proportional to

$$
t=\sin \frac{\pi}{\lambda} \cdot \Delta n \cdot L
$$

Figure $6: I(1 / 2 p)$ proportional to $t^{2}$.

6. Analysis of electric functions. - Real-time tests have been performed on a spectral analysis optical bench developped by MATRA in France [11]. Well known electric functions, such as a sine, a square and a triangle are displayed on the interface by means of the addressing electronics, and their spectral analysis are displayed on a TV screen by means of a vidicon tube and an operating electronics able to display on the TV set the spectral intensity distribution $I(v)$ in the same time.

Figure 7 shows the position of frequencies in a spectrum. We have sampled with a sampling frequency $f_{\mathrm{s}}$ square functions of frequencies $f_{\mathrm{s}} / 2, f_{\mathrm{s}} / 3, \ldots, f_{\mathrm{s}} / n$. It appears in the corresponding spectrum (upper on the photographs) and in their intensity distribution $I(v)$

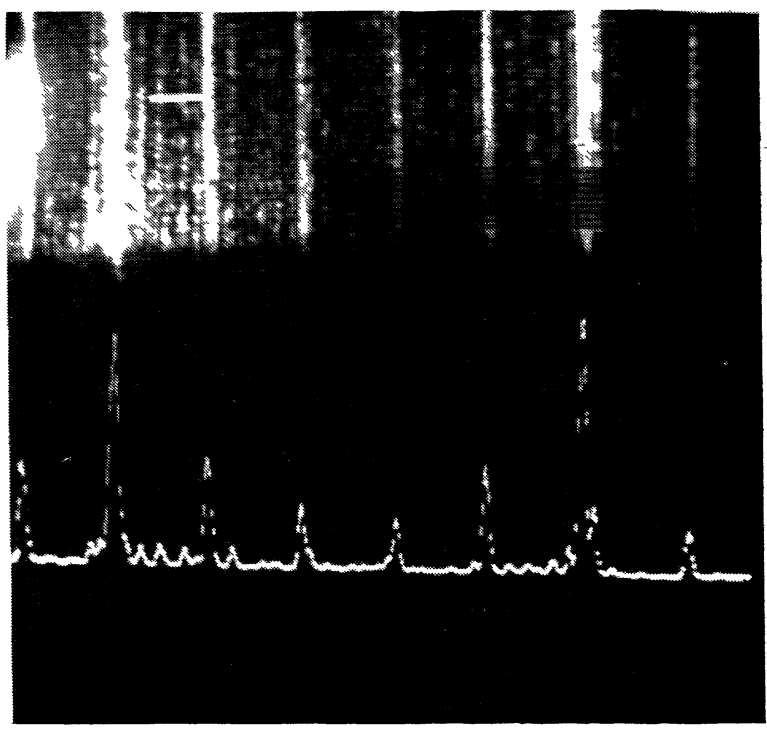

(c)

FIG. 8. - Analyzing various functions of frequency $\left.f_{\mathrm{s}} / 5: a\right)$ sine $\left.\left(2 \pi \frac{f_{\mathrm{s}} t}{5}\right) ; b\right)$ triangle $\left.\left(2 \pi \frac{f_{\mathrm{s}} t}{5}\right) ; c\right)$ square $\left(2 \pi \frac{f_{\mathrm{s}} t}{5}\right)$.

top : diffraction pattern in the focal plane.

bottom : corresponding spectral distribution. 
(lower), between the « 0 » frequency (on the left) and the sampling frequency $1 / p$ (on the right), $(n-1)$ peaks of light diffraction, corresponding to characteristic frequencies, with $n$ equal spaces.

Figure 8 shows the expected difference between the spectrum of functions sine, triangle and square of frequency $f_{\mathrm{s}} / 5$, respectively on the upper, lower left and lower right part of the figure.

Figure 9 gives proof of the linearity of the optical interface : a sine function of frequency $f_{\mathrm{s}} / 5$ and of variable amplitude is displayed. It can be seen from the figure that the light intensity diffracted is proportional to the square amplitude of the sine function displayed. This amplitude is decreasing from 1 (upper) to $1 / \sqrt{2}$ (lower left) and $\frac{1}{2}$ (lower right). The corresponding light intensities in the spectrum are in the square ratios with an accuracy of about $5 \%$.

Figure 10 gives an idea of the spectral resolution achievable with this device. A sine function of frequency $f_{\mathrm{s}} / 32$ (upper) is easily solved. Moreover, by measuring in the spectrum the position of $f_{\max }=f_{\mathrm{s}} / 2$ and the peak width $\Delta f$, we can estimate a resolution of about 30 as the ratio $f_{\max } / \Delta f$, which is quite good for a 64 samples interface.

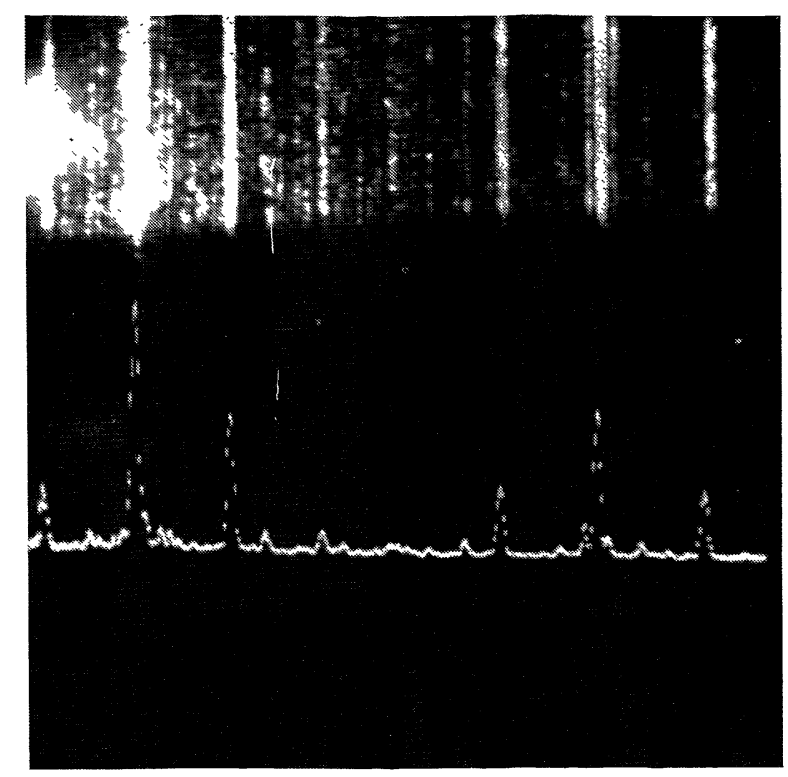

(a)

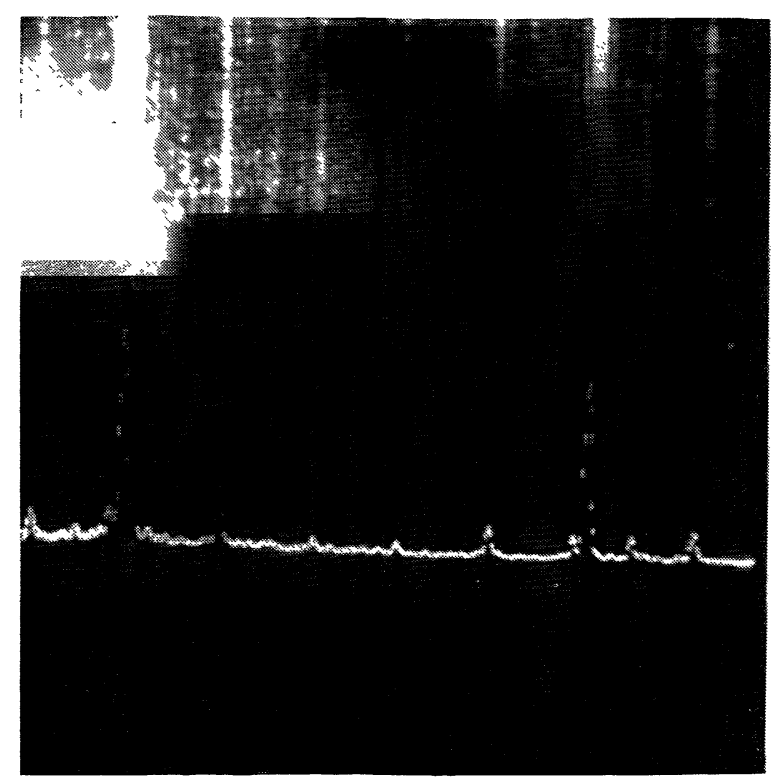

(b)

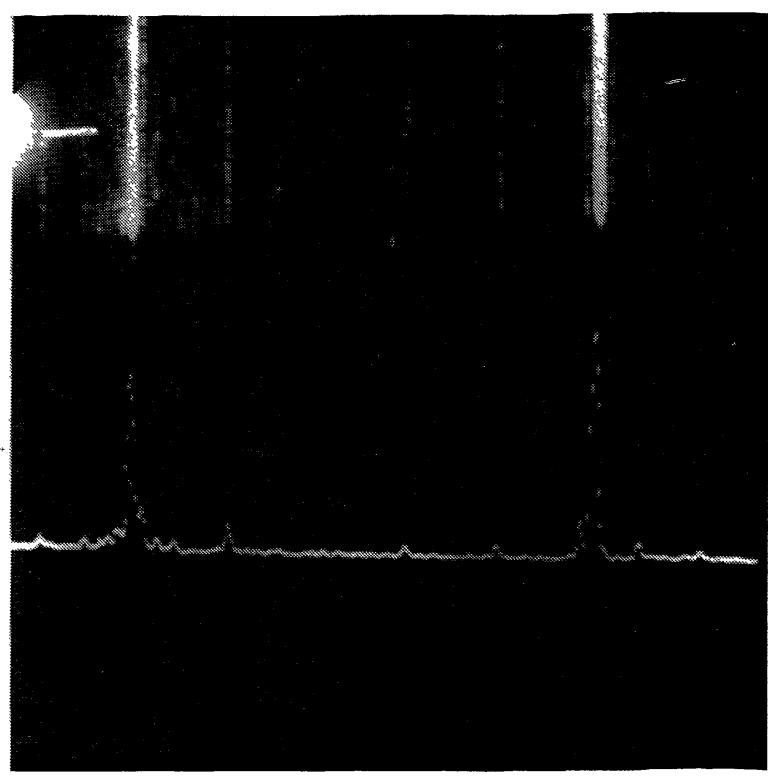

(c)

FIG. 9. - Analyzing $\sin \left(2 \pi \frac{f_{\mathrm{s}} t}{5}\right)$ with a variable amplitude : a) Amplitude $\left.a ; b\right)$ amplitude $\left.a / \sqrt{2} ; c\right)$ amplitude $a / 2$. top : diffraction pattern in the focal plane. bottom : corresponding spectral distribution. 


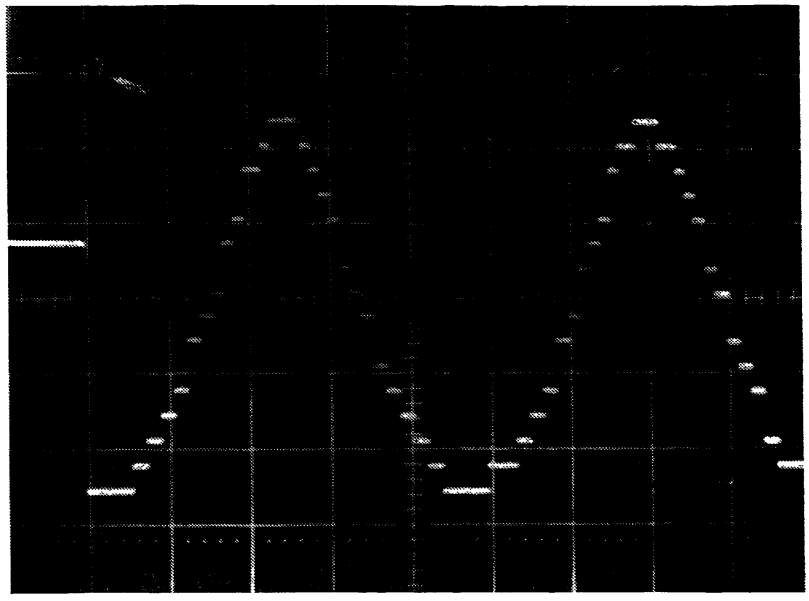

(a)

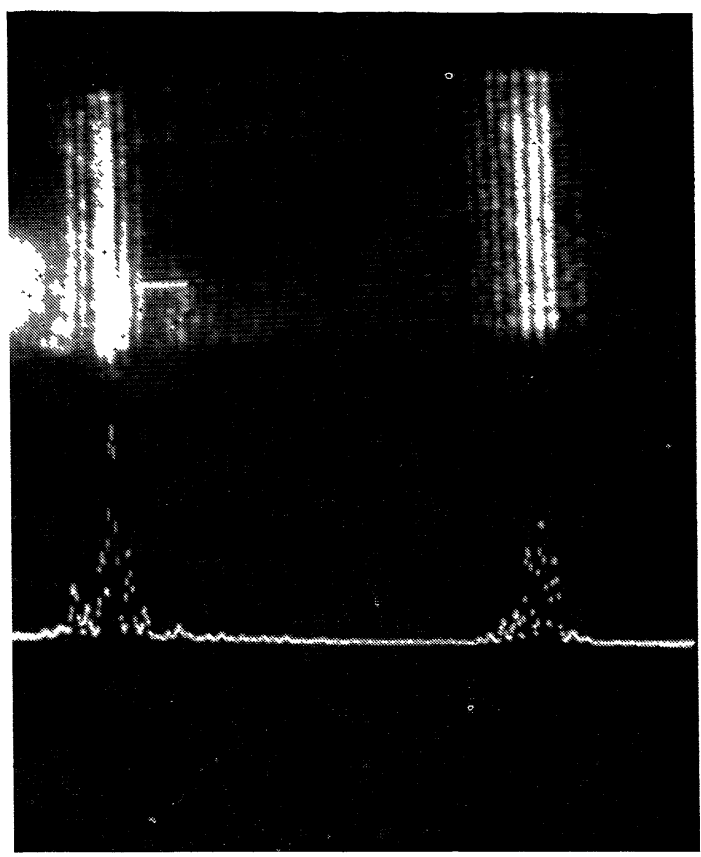

(b)

FIG. 10. - a) Sampled and quantised function $\sin \left(2 \pi \frac{f_{\mathrm{s}} t}{32}\right)$. b) Its spectral analysis.

top : diffraction pattern in the focal plane. bottom : corresponding spectral distribution.
7. Conclusion. - Table I gives the main characteristics of the presented analog electro-optical interface. It must be pointed out that the dynamic range of frequency analysis extends from $2 \mathrm{~Hz}$ to $50 \mathrm{kHz}$ in several steps, the dynamics being equal to 30 in a single step. An accuracy better than $10 \%$ in the intensity of each frequency in the spectrum is caused partly by quantising the analog levels, and partly by the linear approximation in the electronic addressing system.

TABle I. - Main features of the N. L. C. analog interface

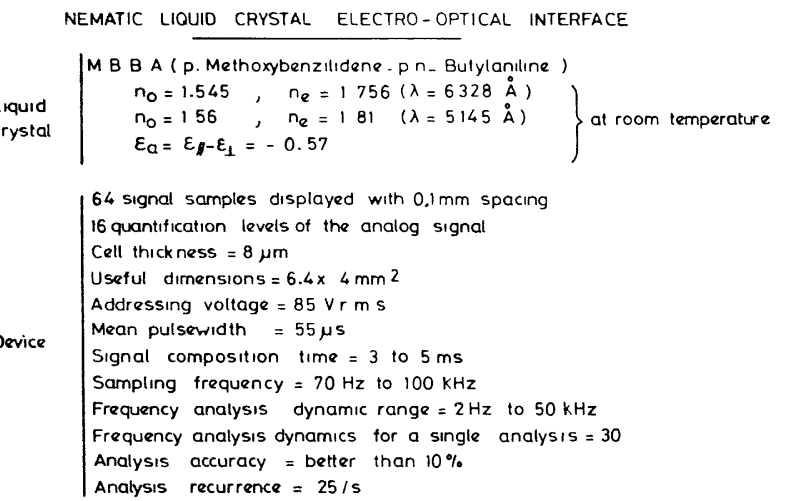

We think that such a device is an example of what can be done with nematic liquid crystals used in the field effect mode, for displaying analog light levels in near realtime.

This interface can be used for spectral analysis of low and medium frequencies signals (ranging from $2 \mathrm{~Hz}$ to $50 \mathrm{kHz}$ ), such as electroencephalogram in medicine, engine supervision in mechanics, and military SONAR signals. It is about to be applied in such coherent optical processes as convolution or crosscorrelation of signals.

Acknowledgments. - Thanks are due to S. Valette for his participation in designing the device.

This work has been partly supported by the Direction des Recherches et Moyens d'Essais in France.

\section{References}

[1] Stroke, G. W., An introduction to coherent optics and holography (Academic Press, New-York) 1966.

[2] Goodman, J. W., Introduction à l'optique de Fourier et à l'holographie (Masson, Paris) 1972.

[3] Grenot, M., Pergrale, J., Donjon, J., Marie, G., Appl. Phys. Lett. 21 (1972) 83.

Donjon, J., Dumont, F., Grenot, M., Hazan, J. P., Marie, G., Pergrale, J., IEEE Trans. El. Dev. E. D. 20 (1973) 1037.

[4] Smith, W. D., Land, C. E., Appl. Phys. Lett. 20 (1972) 169.

[5] Frappier, M., et al., Nouv. Revue Opt. 4 (1971) 221.

[6] Margerum, J. D. et al., Appl. Phys. Lett. 19 (1971) 216.
[7] Feinleib, J., Oliver, D. S., Appl. Opt. 11 (1972) 2752.

[8] Nisenson, P., Isawa, S., Appl. Opt. 11 (1972) 2760.

[9] Cutrona, L. J., Leith, E. N., Palermo, C. J., Porcello, L. J., IRE Trans. Inform. Theory 6 (1960) 386.

[10] Labrunie, G., Robert, J., Borel, J., Revue Phys. Appl. 10 (1975) 143.

[11] ENARD, D., Rapport de synthèse DRME (avril 1972).

Enard, D. et Pouleau, J., Journées d'Etudes du CNES sur le traitement des images (13-15 décembre 1972, Paris), p. 173 du Recueil des exposés publié par le CNES (dépt. des affaires universitaires, 129, rue de l’Université, 75327 Paris Cedex 07). 